\title{
Anomaly of zero-bias conductance peaks in ferromagnet/d-wave superconductor junctions
}

\author{
Z. C. Dong \\ National Laboratory of Solid State Microstructures, Nanjing University, Nanjing 210093, China \\ and Department of Physics, Huaiyin Teachers College, Huaiyin 223001, China \\ D. Y. Xing and Z. D. Wang \\ Department of Physics, University of Hong Kong, Hong Kong \\ and National Laboratory of Solid State Microstructures, Nanjing University, Nanjing 210093, China \\ Ziming Zheng and Jinming Dong \\ National Laboratory of Solid State Microstructures and Department of Physics, Nanjing University, Nanjing 210093, China
}

(Received 8 September 2000; published 22 March 2001)

\begin{abstract}
The Blonder-Tinkham-Klapwijk approach is applied to studying spin-polarized quasiparticle transport in ferromagnet (FM) $/ d$-wave superconductor (SC) junctions by taking into account the roughness of the interfacial barrier, broken time-reversal symmetry (BTRS) states near the surface of the SC, and exchange interactions in the FM. It is shown that (1) the exchange splitting in the FM decreases the height of the zero-bias conductance peak (ZBCP) and may induce a zero-bias conductance dip (ZBCD), (2) the presence of the BTRS states in the SC may make the ZBCP split into two peaks, and (3) the interface roughness obstructs the ZBCP splitting and decreases the height of the $\mathrm{ZBCP}$. The calculated results can account for the $\mathrm{ZBCD}$ observed experimentally in $\mathrm{La}_{2 / 3} \mathrm{Ba}_{1 / 3} \mathrm{MnO}_{3} / \mathrm{DyBa}_{2} \mathrm{Cu}_{3} \mathrm{O}_{7}$ and $\mathrm{La}_{2 / 3} \mathrm{Ba}_{1 / 3} \mathrm{MnO}_{3} / \mathrm{YBa}_{2} \mathrm{Cu}_{3} \mathrm{O}_{7-\delta}$ junctions.
\end{abstract}

DOI: 10.1103/PhysRevB.63.144520

PACS number(s): 74.80.Fp, 74.50.+r, 74.76.Bz

The tunneling spectroscopy in normal-metal/ superconductor (NM/SC) tunnel junctions can provide useful information on the superconducting mechanism and gap. It was indicated theoretically that the unconventional $d$-wave symmetry could lead to phenomena such as midgap surface states $^{1}$ and a zero-bias conductance peak ${ }^{2}$ (ZBCP) due to the sign change in the pair potential. Observations of a ZBCP in the in-plane conductance were reported for NM/SC tunnel junctions on oriented high- $T_{c}$ superconducting films such as $\mathrm{YBa}_{2} \mathrm{Cu}_{3} \mathrm{O}_{7-\delta},{ }^{2-7} \quad \mathrm{Tl}_{2} \mathrm{Ba}_{2} \mathrm{CaCu}_{2} \mathrm{O},{ }^{6} \mathrm{Bi}_{2} \mathrm{Sr}_{2} \mathrm{CaCu}_{2} \mathrm{O}_{8+\delta}$, and $\mathrm{La}_{1.85} \mathrm{Sr}_{0.15} \mathrm{CuO}_{4}$ (Ref. 7) films. The peak height in the experiment was found to be somewhat lowered compared with that in the theory; the agreement in the peak height was suggested to be improved by taking account of impurity scattering and interface roughness in the calculation. ${ }^{2}$ It is highly desirable to perform a serious calculation on the interface roughness effect on the $\mathrm{ZBCP}{ }^{8}$ On the other hand, it was reported that when a magnetic field of a few tesla was applied, some of the ZBCP's split into two peaks, while some of them only broadened without splitting. ${ }^{2}$ Further, a ZBCP splitting of a few meV was observed at low temperatures in zero field. ${ }^{9}$ The splitting of the ZBCP was attributed to the presence of a spontaneously broken time-reversal symmetry phase in the surface state of a $d_{x^{2}-y^{2}}$ state. ${ }^{10}$ However, the presence of the ZBCP splitting at zero field and the absence of the splitting in some junctions under applied magnetic fields are yet to be understood theoretically.

Recently, transport properties in hybrid structures between ferromagnet (FM) and high- $T_{c}$ SC's have received considerable theoretical and experimental attention. ${ }^{11-16}$ For a $\mathrm{La}_{2 / 3} \mathrm{Ba}_{1 / 3} \mathrm{MnO}_{3} / \mathrm{DyBa}_{2} \mathrm{Cu}_{3} \mathrm{O}_{7}$ junction, a zero-bias conductance dip (ZBCD) was observed at zero field and low temperatures, and was attributed to a suppression of Andreev reflection $^{17}(\mathrm{AR})$ as a consequence of high spin polarization in the half metallic FM. ${ }^{15}$ Very recently, the differential conductance in $\mathrm{La}_{2 / 3} \mathrm{Ba}_{1 / 3} \mathrm{MnO}_{3} / \mathrm{YBa}_{2} \mathrm{Cu}_{3} \mathrm{O}_{7-\delta}$ junctions was detected by Sawa et al., ${ }^{16}$ exhibiting a small central peak in the ZBCD. The tunneling spectroscopy of the $\mathrm{FM} / d$-wave SC junctions were analyzed theoretically. ${ }^{18-20}$ Qualitative features in the zero-bias conductance may be reproduced, the effects of the exchange interactions in the FM or/and in the barrier, the barrier height, and the Fermi wave-vector mismatch between the FM and SC regions have also been studied.

In this paper, we extend the Blonder-Tinkham-Klapwijk (BTK) theory, ${ }^{21}$ which was previously used to calculate differential conductance of an $\mathrm{NM} / s$-wave SC junction, to study that of an $\mathrm{FM} / d$-wave SC junction by taking into account the broken time-reversal symmetry (BTRS) states and the barrier roughness. The former is considered by introducing an $s$-wave component near the surface of the $d$-wave SC, forming a mixed $(d+i s)$-wave state. ${ }^{10,22}$ The rough barrier is described by a complex $\delta$-function potential with the real and imaginary prefactors indicating the barrier strength and roughness, respectively. It is shown that the BTRS states may give rise to a splitting of the $\mathrm{ZBCP}$, while the barrier roughness plays a role in obstructing the splitting of the $\mathrm{ZBCP}$. As a result, whether or not the ZBCP splits into two peaks is determined by a competition between the above two factors. Both the exchange splitting in the FM and the interface roughness result in a decrease in height of the ZBCP. In the case of the half metallic FM, the ZBCD behavior observed in experiments can be reproduced by taking account of the interface roughness effects.

Consider an FM/SC junction structure of semi-infinite FM and SC films separated by a very thin insulating film at $x$ $=0$. As shown in Fig. 1, there are three regions: an FM region of $x<0$, a bulk SC region of $x>L$ with a pure $d$-wave 


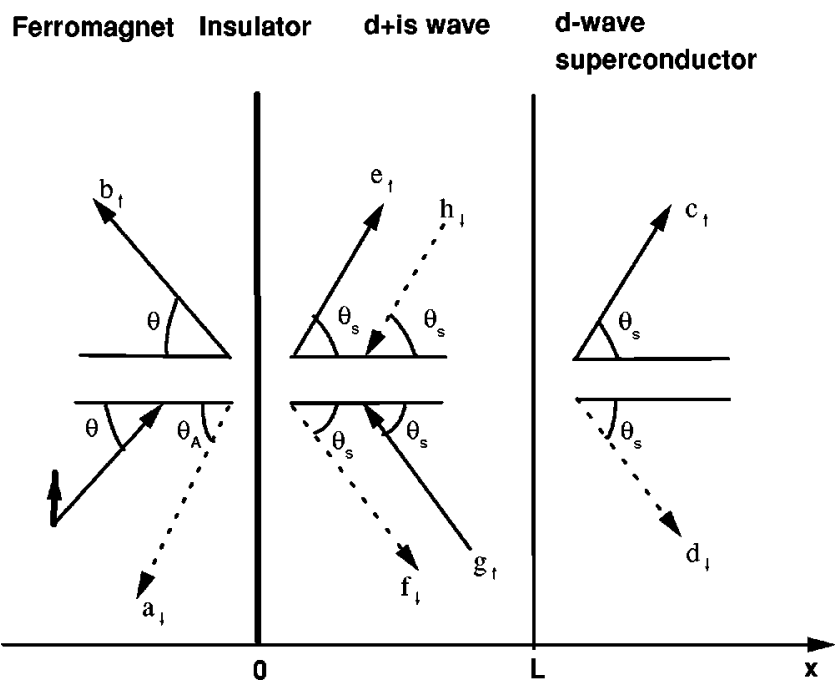

FIG. 1. Schematic illustration of reflections and transmissions of quasiparticles in the FM/SC junction.

order parameter, and a surface SC region of $0<x<L$ with a $(d+i s)$-wave state in which the time-reversal symmetry is locally broken. As is well understood, an inhomogeneity in a $d$-wave SC will induce an $s$-wave component, such as the surface of a $d$-wave $\mathrm{SC}$ and the $\mathrm{FM} / d$-wave $\mathrm{SC}$ interface under consideration here. ${ }^{10,22,23}$ Owing to proximity effects of the FM/SC structure, a BTRS state must appear at the surface SC region of thickness at least equal to the superconducting coherence length $\xi$. Such a BTRS state should be assumed to be the $(d+i s)$-wave state, and $L$ to be of the order of $\xi$. The pair potential takes different forms in the three regions. $\Delta(x)=0$ for $x<0$. For $x>L, \Delta(x)=\Delta_{ \pm}^{d}$ $=\Delta_{0} \cos \left(2 \theta_{s} \mp 2 \alpha\right)$, where $\Delta_{+}^{d}$ and $\Delta_{-}^{d}$ stand for the pair potentials for electronlike and holelike quasiparticles, respectively, $\alpha$ is the angle between the $a$ axis of the crystal and the interface normal, and $\theta_{s}$ has been indicated in Fig. 1. For 0 $<x<L, \Delta(x)=\Delta_{ \pm}^{m}=\Delta_{d} \cos \left(2 \theta_{s} \mp 2 \alpha\right)+i \Delta_{s}$, where $\Delta_{d}$ and $\Delta_{s}$ are the magnitude of the $d$ - and $s$-wave components of the pair potential, respectively. It is worth noting that the effective pair potentials experienced by the electronlike and holelike excitations in the $d$-wave SC are usually different and may even have opposite signs under certain circumstances.

We adopt the Bogoliubov-de Gennes (BdG) approach $^{24}$ to study the FM/SC junction. Within the Stoner model, the motion of conduction electrons inside the FM can be described by an effective single-particle Hamiltonian with an exchange interaction. In the absence of spin-flip scattering, the spindependent (four-component) $\mathrm{BdG}$ equations are decoupled into two sets of (two-component) equations: one for the spin-up electronlike and spin-down holelike quasiparticle wave functions $\left(u_{\uparrow}, v_{\downarrow}\right)$, the other for $\left(u_{\downarrow}, v_{\uparrow}\right) .{ }^{18}$ The BdG equation for $\left(u_{\uparrow}, v_{\downarrow}\right)$ is given by

$$
\left[\begin{array}{cc}
H_{0}(\mathbf{r})-h(\mathbf{r}) & \Delta(x, \theta) \\
\Delta^{\star}(x, \theta) & -H_{0}(\mathbf{r})^{\star}-h(\mathbf{r})
\end{array}\right]\left[\begin{array}{l}
u_{\uparrow}(x, \theta) \\
v_{\downarrow}(x, \theta)
\end{array}\right]=E\left[\begin{array}{l}
u_{\uparrow}(x, \theta) \\
v_{\downarrow}(x, \theta)
\end{array}\right] .
$$

Here $H_{0}(\mathbf{r})=-\hbar^{2} \nabla_{\mathbf{r}}^{2} / 2 m+V(\mathbf{r})-E_{F}$ with $V(\mathbf{r})$ the usual static potential, and $h(\mathbf{r})=h_{0} \Theta(-x)$ with $h_{0}$ the exchange energy in the FM and $\boldsymbol{\Theta}(x)$ the unit step function. The excitation energy $E$ is measured relative to the Fermi energy $E_{F}$. For the $d$ - or $(d+i s)$-wave SC, both $u_{\uparrow}$ and $v_{\downarrow}$ as well as $\Delta$ are angle dependent.

In the presence of interface roughness, the FM/SC interface barrier at $x=0$ can be described by a $\delta$-type potential $\delta(x)$ with a random roughness function $g(y, z)$ so that the barrier potential is given by $U(\mathbf{r})=U \delta(x) g(y, z)$. In the Green's function approach under the "white noise" approximation, the self-energy contains an imaginary part independent of momentum. It then follows that the interface barrier may be modeled by an effective interface potential ${ }^{25}$

$$
U \delta(x)=\left(U_{0} \hat{1}-i P \hat{\tau}_{3}\right) \delta(x),
$$

where $\hat{1}$ is the unit matrix and $\hat{\tau}_{3}$ the Pauli matrix. In this effective potential, $U_{0}$ indicates the barrier strength and $P$ describes the scattering effect during tunneling through the rough barrier.

Consider a beam of spin-up electrons incident on the interface at $x=0$ from the FM at an angle $\theta$ to the interface normal. As shown in Fig. 1, there are four possible trajectories: normal reflection $\left(b_{\uparrow}\right)$, Andreev reflection $\left(a_{\downarrow}\right)$, transmission to the $\mathrm{SC}$ as electronlike quasiparticles $\left(e_{\uparrow}\right)$, and transmission as holelike quasiparticles $\left(f_{\downarrow}\right)$. We wish to point out here that the AR coefficient $a_{\downarrow}$ is labeled with subscript $\downarrow$ because the AR results in an electron deficiency in the spin-down subband of the FM, even though it is at times called a spin-up hole. With general solutions of the $\mathrm{BdG}$ equation (1), the wave functions in three regions are described by

$$
\Psi_{I}=\left(\begin{array}{l}
1 \\
0
\end{array}\right) e^{i q_{\uparrow} x \cos \theta}+a_{\downarrow}\left(\begin{array}{l}
0 \\
1
\end{array}\right) e^{i q_{\downarrow} x \cos \theta_{A}}+b_{\uparrow}\left(\begin{array}{l}
1 \\
0
\end{array}\right) e^{-i q_{\uparrow} x \cos \theta}
$$

for $x<0$,

$$
\begin{aligned}
& \Psi_{I I}=e_{\uparrow}\left(\begin{array}{c}
u_{+}^{m} e^{i \phi_{+}^{m}} \\
v_{+}^{m}
\end{array}\right) e^{i k_{+}^{m} x \cos \theta_{s}}+f_{\downarrow}\left(\begin{array}{c}
v_{-}^{m} e^{i \phi_{-}^{m}} \\
u_{-}^{m}
\end{array}\right) e^{-i k_{-}^{m} x \cos \theta_{s}}
\end{aligned}
$$

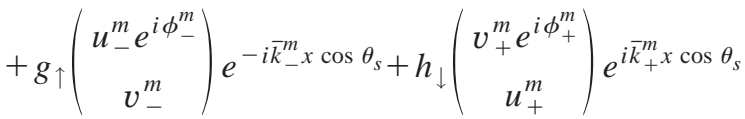

for $0<x<L$, and



for $\quad x>L$. Here $\quad q_{\uparrow} \simeq \sqrt{2 m\left(E_{F}+h_{0}\right) / \hbar^{2}} \quad$ and $\quad q_{\downarrow}$ $\simeq \sqrt{2 m\left(E_{F}-h_{0}\right) / \hbar^{2}}$, indicating different Fermi wave vectors for the spin-up and spin-down subbands in the FM. In the surface $\mathrm{SC}$ region, $k_{ \pm}^{m}=k_{F}\left[1 \pm \sqrt{E^{2}-\left|\Delta_{ \pm}^{m}\right|^{2}} / E_{F}\right]^{1 / 2}$ and $\bar{k}_{ \pm}^{m}=k_{F}\left[1 \mp \sqrt{E^{2}-\left|\Delta_{ \pm}^{m}\right|^{2}} / E_{F}\right]^{1 / 2}$; and in the bulk SC region, 
$k_{+}^{d}=k_{F}\left[1 \pm \sqrt{E^{2}-\left|\Delta_{+}^{d}\right|^{2}} / E_{F}\right]^{1 / 2}$, where $k_{F}=\sqrt{2 m E_{F} / \hbar^{2}}$ is the Fermi wave vector in the SC. All the wave vectors in Eqs. (3b) and (3c), $k_{ \pm}^{m}, \bar{k}_{ \pm}^{m}$ and $k_{ \pm}^{d}$, are approximately equal to $k_{F} \cdot\left(u_{ \pm}^{m}\right)^{2}=1-\left(v_{ \pm}^{m}\right)^{2}=\frac{1}{2}\left(1+\sqrt{1-\left|\Delta_{ \pm}^{m} / E\right|^{2}}\right)$ and $\left(u_{ \pm}^{d}\right)^{2}$ $=1-\left(v_{ \pm}^{d}\right)^{2}=\frac{1}{2}\left(1+\sqrt{1-\left|\Delta_{ \pm}^{d} / E\right|^{2}}\right)$. In Eqs. (3b) and (3c), $\phi_{ \pm}^{m}$ and $\phi_{ \pm}^{d}$ are given by

$$
\begin{gathered}
\phi_{ \pm}^{m}=\cos ^{-1}\left[\frac{\cos 2\left(\theta_{s} \mp \alpha\right)}{\sqrt{\cos ^{2} 2\left(\theta_{s} \mp \alpha\right)+\left(\Delta_{s} / \Delta_{d}\right)^{2}}}\right], \\
\phi_{ \pm}^{d}=\cos ^{-1}\left[\frac{\cos 2\left(\theta_{s} \mp \alpha\right)}{\left|\cos 2\left(\theta_{s} \mp \alpha\right)\right|}\right] .
\end{gathered}
$$

In the FM, $q_{\uparrow}$ is greater than $q_{\downarrow}$ due to the presence of the exchange splitting $2 h_{0}$. Also, neither of them is equal to $k_{F}$ in the SC. However, they must satisfy the condition $q_{\uparrow} \sin \theta=q_{\downarrow} \sin \theta_{A}=k_{F} \sin \theta_{S}$ to guarantee the conservation of the momentum components parallel to the interface. As a result, $\theta, \theta_{A}$, and $\theta_{s}$ differ from each other except when $\theta$ $=0$; in the present case, $q_{\uparrow}>k_{F}>q_{\downarrow}$ so that $\theta_{A}<\theta_{S}<\theta$. With increasing $\theta$, both $\theta_{A}$ and $\theta_{s}$ become large. As $\theta$ exceeds $\sin ^{-1}\left(q_{\downarrow} / q_{\uparrow}\right)$, the $x$ component of the wave vector in the AR process $\sqrt{q_{\downarrow}^{2}-q_{\uparrow}^{2} \sin ^{2} \theta}$ will become purely imaginary so that the Andreev reflected quasiparticles do not propagate, and they were referred to as virtual AR. Further, when $\theta$ $>\sin ^{-1}\left(k_{F} / q_{\uparrow}\right)$, the transmitted quasiparticles do not propagate and so the net charge current from the FM to SC vanishes.

All coefficients in Eqs. (3a)-(3c) can be determined by boundary conditions at $x=0$ and $x=L$. They are $\psi_{I I}(0)$ $=\psi_{I}(0), \quad\left(d \psi_{I I} / d x\right)_{x=0}-\left(d \psi_{I} / d x\right)_{x=0}=2 m U \psi_{I}(0) / \hbar^{2}$, $\psi_{I I}(L)=\psi_{I I I}(L)$, and $\left(d \psi_{I I} / d x\right)_{x=L}=\left(d \psi_{I I I} / d x\right)_{x=L}$. Using the boundary conditions on the wave functions and carrying out a little tedious algebra, we find

$$
\begin{gathered}
a_{\downarrow}=4 r_{\uparrow} \bar{u}_{-} \bar{v}_{+} e^{-i \phi_{+}^{m}} / D, \\
b_{\uparrow}=\left\{\left[2\left(i Z_{1}+Z_{2}\right)-r_{\uparrow}-1\right]\left[2\left(i Z_{1}-Z_{2}\right)-r_{\downarrow}+1\right] \bar{v}_{-}\right. \\
\times \bar{v}_{-} e^{i\left(\phi_{-}^{m}-\phi_{+}^{m}\right)}-\left[2\left(i Z_{1}+Z_{2}\right)-r_{\uparrow}+1\right] \\
\left.\times\left[2\left(i Z_{1}-Z_{2}\right)-r_{\downarrow}-1\right] \bar{u}_{+} \bar{u}_{-}\right\} / D,
\end{gathered}
$$

with

$$
\begin{gathered}
D=\left[2\left(i Z_{1}+Z_{2}\right)+r_{\uparrow}+1\right]\left[2\left(i Z_{1}-Z_{2}\right)-r_{\downarrow}-1\right] \bar{u}_{+} \bar{u}_{-} \\
-\left[2\left(i Z_{1}+Z_{2}\right)+r_{\uparrow}-1\right]\left[2\left(i Z_{1}-Z_{2}\right)\right. \\
\left.-r_{\downarrow}+1\right] \bar{v}_{+} \bar{v}_{-} e^{i\left(\phi_{-}^{m}-\phi_{+}^{m}\right)} \\
\bar{u}_{ \pm}=u_{ \pm}^{m}-Q_{ \pm} v_{ \pm}^{m} \\
\bar{v}_{ \pm}=v_{ \pm}^{m}-Q_{ \pm} u_{ \pm}^{m} \\
Q_{+}=\frac{v_{+}^{m} u_{+}^{d} e^{i\left(\phi_{-}^{m}-\phi_{+}^{m}\right)}-u_{+}^{m} v_{+}^{d}}{u_{+}^{m} u_{+}^{d} e^{i\left(\phi_{-}^{m}-\phi_{+}^{m}\right)}-v_{+}^{m} v_{+}^{d}} \exp \left[i\left(k_{+}^{m}-\bar{k}_{+}^{m}\right) L \cos \theta_{s}\right]
\end{gathered}
$$

$$
Q_{-}=\frac{u_{-}^{m} v_{-}^{d} e^{i\left(\phi_{-}^{d}-\phi_{-}^{m}\right)}-v_{-}^{m} u_{-}^{d}}{v_{-}^{m} v_{-}^{d} e^{i\left(\phi_{-}^{d}-\phi_{-}^{m}\right)}-u_{-}^{m} u_{-}^{d}} \exp \left[-i\left(k_{-}^{m}-\bar{k}_{-}^{m}\right) L \cos \theta_{s}\right]
$$

Here $\quad r_{\uparrow}=q_{\uparrow} \cos \theta /\left(k_{F} \cos \theta_{s}\right), \quad r_{\downarrow}=q_{\downarrow} \cos \theta_{A} /\left(k_{F} \cos \theta_{s}\right), Z_{1}$ $=Z_{10} / \cos \theta_{s}$, and $Z_{2}=Z_{20} / \cos \theta_{s}$ with $Z_{10}=m U_{0} / \hbar^{2} k_{F}$ and $Z_{20}=m P / \hbar^{2} k_{F}$. In Eqs. (5)-(11), all the wave vectors of electronlike and holelike quasiparticles in the two SC regions have been replaced by $k_{F}$ except those appearing in the exponents. For spin-down electrons incident on the interface at $x=0, a_{\uparrow}$ and $b_{\downarrow}$ can be similarly obtained, having symmetric expressions compared with Eqs. (5)-(11). Besides, for an incident electron with spin down, since its wave vectors are always smaller than those of the hole due to $\operatorname{AR}\left(q_{\downarrow}<q_{\uparrow}\right), \theta$ is always greater than $\theta_{A}$ and there is no virtual AR for any incident angle.

The tunneling conductance of an $\mathrm{NM} / s$-wave SC junction has been given by the BTK theory, ${ }^{21}$ with the contribution of AR being included. The BTK approach is readily extended to the spin-dependent transport through an $\mathrm{FM} / d$-wave $\mathrm{SC}$ junction, and the differential conductance is given by ${ }^{20}$

$$
\begin{aligned}
G(\theta)= & G_{\uparrow}+G_{\downarrow}=\frac{2 e^{2}}{h} \operatorname{Re} \sum_{s=\uparrow, \downarrow} \\
& \times P_{s}\left(1+\frac{q_{s}^{-} \cos \theta_{A}}{q_{s} \cos \theta}\left|a_{s}^{-}\right|^{2}-\left|b_{s}\right|^{2}\right),
\end{aligned}
$$

where $P_{\uparrow}=\left(E_{F}+h_{0}\right) / 2 E_{F}$ and $P_{\downarrow}=\left(E_{F}-h_{0}\right) / 2 E_{F}$ are the polarizations in the FM for up and down spins, respectively, and $\bar{s}$ stands for the spin opposite to $s$. In the experiments, the measured conductance is given by a weighted average over contributions from all possible electron trajectories,

$$
G=\frac{1}{2} \int_{-\pi / 2}^{\pi / 2} d \theta \cos \theta G(\theta)
$$

In what follows we calculate the conductance spectrum in terms of Eqs. (12) and (13) together with Eqs. (5)-(11). Let us first study effects of the exchange splitting on the conductance spectrum in the absence of BTRS in the SC and interface roughness by taking $\Delta_{s}=0$ and $Z_{2}=0$. Figure 2(a) shows the normalized conductance $G$ versus scaled energy $E / \Delta_{0}$ for different $h_{0} / E_{F}$. It is found that with increasing the exchange splitting, the $\mathrm{ZBCP}$ is lowered rapidly and evolved gradually into a set of zero-bias conductance dips with surviving central peaks. As $h_{0} / E_{F}$ is increased to 0.999 (i.e., the FM becomes a half metal), the central peak in the ZBCD disappears. The drop of the zero-bias conductance shown in Fig. 2(a) can be attributed to the fact that for a given incident angle, with $h_{0} / E_{F}$ increased beyond a threshold, the AR process for the incident electron with spin up vanishes and only the AR of the spin-down electrons has contributed to the ZBCP. Taking into account the condition $\sin \theta>q_{\downarrow} / q_{\uparrow}$ with $\left(q_{\downarrow} / q_{\uparrow}\right)^{2}=\left(E_{F}-h_{0}\right) /\left(E_{F}+h_{0}\right)$, we get the threshold for $h_{0} / E_{F}$ equal to $\cos ^{2} \theta /\left(1+\sin ^{2} \theta\right)$.

In the presence of BTRS surface states, by taking $L=\xi$ $=\hbar v_{F} / \pi \Delta_{0}$, the calculated results for $\Delta_{s} / \Delta_{d}=0.5$ and 1 are shown in Figs. 2(b) and (c), respectively. Two interesting 

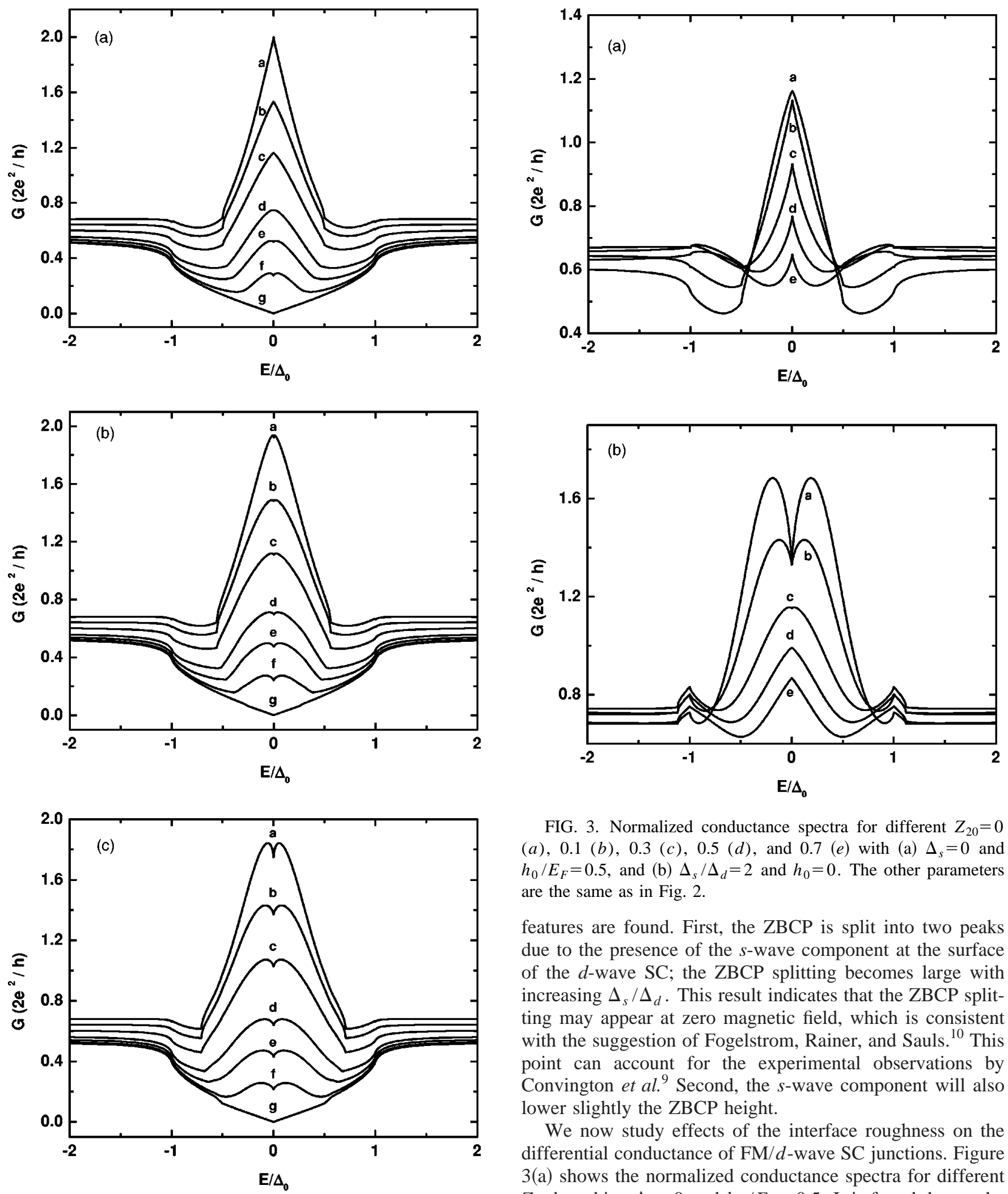

FIG. 3. Normalized conductance spectra for different $Z_{20}=0$ (a), $0.1(b), 0.3(c), 0.5(d)$, and $0.7(e)$ with (a) $\Delta_{s}=0$ and $h_{0} / E_{F}=0.5$, and (b) $\Delta_{s} / \Delta_{d}=2$ and $h_{0}=0$. The other parameters are the same as in Fig. 2.

features are found. First, the ZBCP is split into two peaks due to the presence of the $s$-wave component at the surface of the $d$-wave $\mathrm{SC}$; the ZBCP splitting becomes large with increasing $\Delta_{s} / \Delta_{d}$. This result indicates that the ZBCP splitting may appear at zero magnetic field, which is consistent with the suggestion of Fogelstrom, Rainer, and Sauls. ${ }^{10}$ This point can account for the experimental observations by Convington et al. ${ }^{9}$ Second, the $s$-wave component will also lower slightly the ZBCP height.

We now study effects of the interface roughness on the differential conductance of FM/ $d$-wave SC junctions. Figure 3(a) shows the normalized conductance spectra for different $Z_{20}$ by taking $\Delta_{s}=0$ and $h_{0} / E_{F}=0.5$. It is found that as the interface roughness is increased by increasing $Z_{20}$, the $\mathrm{ZBCP}$ is gradually lowered, and the ZBCD with surviving central peak may appear. From this point, the effect of the interface roughness is similar to that of the exchange splitting in the FM. The difference between them is that the interface roughness has another important effect of resisting 
the splitting of the ZBCP, which can be seen in Fig. 3(b). With $Z_{20}$ increased, the ZBCP splitting due to the BTRS surface states becomes small and disappears gradually. As a result, the interface roughness has at least two effects on the conductance spectra: to resist the splitting of the ZBCP and to lower the height of the ZBCP. From the discussions above, it follows that whether the ZBCP splits into two peaks is mainly determined by the competition between two factors. One is the BTRS due to the $s$-wave component at the surface of the SC, which is favorable to the splitting of the $\mathrm{ZBCP}$; the other is the interface roughness that obstructs the ZBCP splitting, as shown in Fig. 3(b). The suppression effect of the interface roughness on the ZBCP splitting results and the competition between the above two factors may account for inconsistent experimental reports. The ZBCP splitting observed in some NM/SC junctions at zero magnetic field ${ }^{9}$ may be attributed to a strong BTRS and a weak interface roughness, while the absence of the ZBCP splitting in other $\mathrm{NM} / \mathrm{SC}$ junctions even under applied magnetic fields ${ }^{2}$ or in the $\mathrm{FM} / d$-wave SC junctions ${ }^{15,16}$ may arise from the strong suppression effect of rough interfaces. To make a comparison between the theoretical and experimental results, ${ }^{15,16}$ we plot the calculated conductance spectra for a half metallic $\mathrm{FM} / d$-wave SC tunnel junction in Fig. 4 , where $h_{0} / E_{F}$ $=0.999$ and different values of $Z_{20}$ are taken. All the curves exhibit the ZBCD behavior that is evidently a feature of the half metallic FM electrode. In the absence of the interface roughness $\left(Z_{20}=0\right)$, curve $a$ in Fig. 4 corresponds to curve $g$ in Fig. 2(a). A central peak is induced at the ZBCD by the interface roughness and the peak becomes gradually higher with $Z_{20}$ increased. It is found that the experimental curve in Ref. 15 is like curve $a$ in Fig. 4 while that in Ref. 16 is like curve $c$ or $d$, indicating that besides the exchange splitting in the FM, the effect of the interface roughness plays an important role in determining the shape of the conductance spectrum.

In summary we have employed an effective model to describe the roughness of the interfacial barrier and the broken time-reversal symmetry states in $\mathrm{FM} / d$-wave $\mathrm{SC}$ junctions. It is shown that the exchange splitting in the FM and the interface roughness can lead to a decrease of the zero-bias conductance peak, in particular, the former may give rise to a

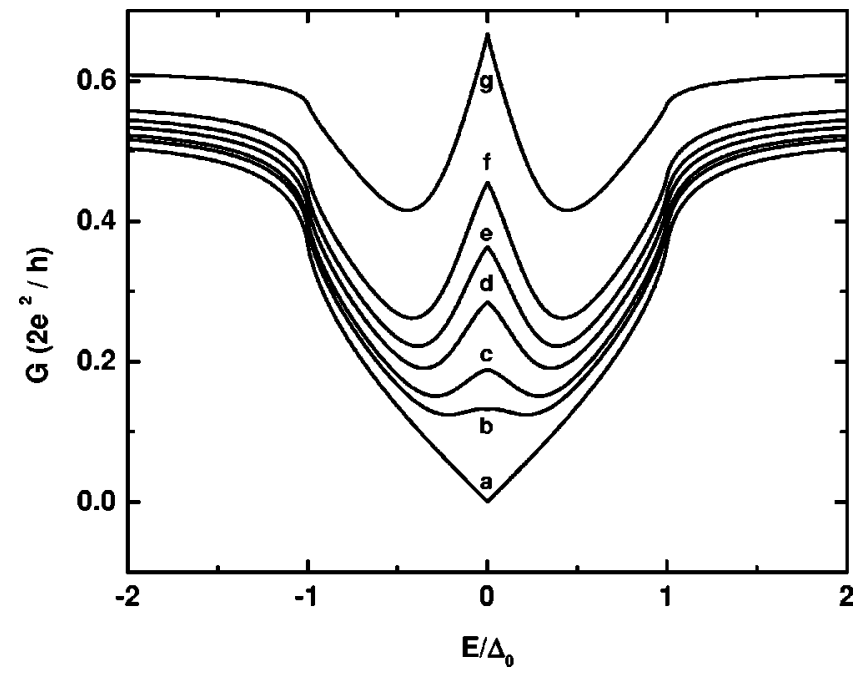

FIG. 4. Normalized conductance spectra for different $Z_{20}=0$ $(a), 0.02(b), 0.03(c), 0.05(d), 0.07(e), 0.1(f)$, and $0.3(g)$ with $h_{0} / E_{F}=0.999$ and $\Delta_{s}=0$. The other parameters are the same as in Fig. 2.

zero-bias conductance dip. Another important result is that the BTRS states in the SC may give rise to the ZBCP splitting, but the interface scattering due to barrier roughness resists it. As a result, whether or not the zero-bias conductance splits is determined by the competition between them. The present calculated results can account for the zero-bias conductance dip observed experimentally in $\mathrm{La}_{2 / 3} \mathrm{Ba}_{1 / 3} \mathrm{MnO}_{3} / \mathrm{DyBa}_{2} \mathrm{Cu}_{3} \mathrm{O}_{7}$ and $\mathrm{La}_{2 / 3} \mathrm{Ba}_{1 / 3} \mathrm{MnO}_{3} / \mathrm{YBa}_{2} \mathrm{Cu}_{3} \mathrm{O}_{7-\delta}$ junctions. In the present model, we have neglected the spatial variation of the pair potential in the SC due to proximity effects and the spin flip of the spin-polarized currents. Inclusion of these effects would be necessary for a complete theory, which merits further study.

This work was supported by a grant of the Research Grant Council of Hong Kong, and a grant for the State Key Program for Basic Research of China, and a grant (No. 19874011) of the National Natural Science Foundation of China.
${ }^{1}$ C. R. Hu, Phys. Rev. Lett. 72, 1526 (1994).

${ }^{2}$ S. Kashiwaya, Y. Tanaka, M. Koyanagi, H. Takashima, and K. Kajimura, Phys. Rev. B 51, 1350 (1995).

${ }^{3}$ T. Walsh, Int. J. Mod. Phys. B 6, 125 (1992).

${ }^{4}$ J. Lesueur, L. H. Greene, W. L. Feldmann, and A. Inam, Physica C 191, 325 (1992).

${ }^{5}$ L. Alff, H. Takashima, S. Kashiwaya, N. Terada, H. Ihara, Y. Tanaka, M. Koyanagi, and K. Kajimura, Phys. Rev. B 55, 14757 (1997).

${ }^{6}$ J. W. Ekim, Y. Xu, S. Mao, T. Venkatesan, D. W. Face, M. Eddy, and S. A. Wolf, Phys. Rev. B 56, 13746 (1997).

${ }^{7}$ L. Alff, A. Beck, R. Gross, A. Marx, S. Kleefisch, Th. Bauch, H. Sato, M. Naito, and G. Koren, Phys. Rev. B 58, 11197 (1998).
${ }^{8}$ Yu. S. Barash, A. A. Svidzinsky, and H. Burkhardt, Phys. Rev. B 55, 15282 (1997).

${ }^{9}$ M. Covington, M. Aprili, E. Paraoanu, L. H. Greene, F. Xu, J. Zhu, and C. A. Mirkin, Phys. Rev. Lett. 79, 277 (1997).

${ }^{10}$ M. Fogelstrom, D. Rainer, and J. A. Sauls, Phys. Rev. Lett. 79, 281 (1997).

${ }^{11}$ R. Meservey and P. M. Tedrow, Phys. Rep. 238, 173 (1994).

${ }^{12}$ M. J. M. de Jong and C. W. J. Beenakker, Phys. Rev. Lett. 74, 1657 (1995).

${ }^{13}$ R. J. Soulen, Jr., J. M. Byers, M. S. Osofsky, B. Nadgorny, T. Ambrose, S. F. Cheng, P. R. Broussard, C. T. Tanaka, J. Nowak, J. S. Moodera, A. Barry, and J. M. D. Coey, Science 282, 85 (1998). 
${ }^{14}$ S. K. Upadhyay, A. Palanisami, R. N. Louie, and R. A. Buhrman, Phys. Rev. Lett. 81, 3247 (1998).

${ }^{15}$ V. A. Vasko, K. R. Nikolaev, V. A. Larkin, P. A. Kraus, and A. M. Goldman, Appl. Phys. Lett. 73, 844 (1998).

${ }^{16}$ A. Sawa, S. Kashiwaya, H. Obara, H. Yamasaki, M. Koyanagi, and Y. Tanaka, Physica B 284-288, 493 (2000).

${ }^{17}$ A. F. Andreev, Zh. Éksp. Teor. Fiz. 46, 1823 (1964) [Sov. Phys. JETP 19, 1228 (1964)].

${ }^{18}$ J. X. Zhu, B. Friedman, and C. S. Ting, Phys. Rev. B 59, 9558 (1999).

${ }^{19}$ I. Zutic and O. T. Valls, Phys. Rev. B 60, 6230 (1999); 61, 1555 (2000).

${ }^{20}$ S. Kashiwaya, Y. Tanaka, N. Yoshida, and M. R. Beasley, Phys.
Rev. B 60, 3572 (1999).

${ }^{21}$ G. E. Blonder, M. Tinkham, and T. M. Klapwijk, Phys. Rev. B 25, 4515 (1982).

${ }^{22}$ M. Matsumoto and H. Shiba, J. Phys. Soc. Jpn. 64, 3384 (1995); 64, 4867 (1995); 65, 2194 (1996).

${ }^{23}$ M. Sigrist, D. B. Bailey, and R. B. Laughlin, Phys. Rev. Lett. 74, 3249 (1995); D. B. Bailey, M. Sigrist, and R. B. Laughlin, Phys. Rev. B 55, 15239 (1997).

${ }^{24}$ P. G. de Gennes, Superconductivity of Metals and Alloys (Benjamin, New York, 1966).

${ }^{25}$ Z. C. Dong, D. Y. Xing, Z. D. Wang, and L. Sheng, Z. Phys. B: Condens. Matter 100, 329 (1996); J. X. Zhu, Z. D. Wang, D. Y. Xing, and Z. C. Dong, Physica C 260, 216 (1996). 